\title{
¿POr Qué Pensar sobre el hábitat desDe la ARQUITECTURA EN EL SUR Y DESDE EL SUR GLOBAL?
}

\author{
Why to think about the habitat from the architecture in the south \\ and from the global south?
}

\author{
Adriana Inés Olivares González \\ Fecha de recepción: 11-06-2019 - Fecha de aceptación: 23-07-2019 \\ Hábitat y Sociedad (ISSN 2173-125X), n. ${ }^{\circ}$ 12, noviembre de 2019, pp. 115-122. \\ http://dx.doi.org/10.12795/HabitatySociedad.2019.i12.07
}

\section{Summary}

The book Visiones del Hábitat de América Latina comprises a set of ideas about the processes that have characterized the production of social housing in Latin America in the last fifty years with a South to South perspective. This book which was coordinated by Manuel Martín Hernández and Vicente Díaz García, integrates the perspective of renowned specialists with different backgrounds who have devoted their research and professional activity to the study of social housing as one of the main elements defining the quality of people's lives and therefore their dignity as human beings.

Most of the authors were gathered together for the first time thanks to the HABYTED Program (Technology for Social Housing Program) in 1987; among them we have Julián Salas, Víctor Pelli, Carlos González Lobo, Esteban de Manuel Jerez, Aurelio Ferrero, Gustavo Romero y Vicente Díaz. Some have led organizations well known for their critical view towards the production of social housing, for example Joan MacDonald. Others have met in specialized university academic events: Aurelio Ferrero, María Bernabela Pelli and Raúl Vallés; or in postgraduate programs: Daniel González Romero, Jorge Pérez Jaramillo and Guillermo Gaete.

All of them, assemble the experience of the cities in the Global South. They set out realities that, except for few cases and isolated projects, have been poorly considered in the construction of public policies regarding housing in the countries of the region. However, these are necessary experiences to write a new chapter on habitat from the South.

\section{Key words}

Habitat; Architecture; Right to the city; Social participation; Global South

\section{Resumen}

El libro Visiones del Hábitat de América Latina comprende un conjunto de ideas sobre los procesos que han caracterizado la producción de vivienda social en América Latina en los últimos cincuenta años desde una visión Sur-Sur. Coordinado por Manuel Martín Hernández y Vicente Díaz García, este libro integra la mirada de reconocidos especialistas de diversa factura que han dedicado su actividad investigativa y/o profesional al estudio de la vivienda social como uno de los elementos mas importantes que define la calidad de vida de las personas y, por lo tanto, su dignidad como seres humanos.

Una buena parte de los autores fueron reunidos por primera vez gracias al programa HABYTED en 1987 como Julián Salas, Víctor Pelli, Carlos González Lobo, Esteban de Manuel Jerez, Aurelio Ferrero, Gustavo Romero y Vicente Díaz; otros han liderado organizaciones reconocidas por su postura critica respecto de la producción social del hábitat como Joan MacDonald; otros más coincidieron en eventos académicos universitarios especializados: Aurelio Ferrero, María Bernabela Pelli y Raúl Vallés; o en programas de posgrado: Daniel González Romero, Jorge Pérez Jaramillo y Guillermo Gaete.

Todos ellos articulan la experiencia de ciudades del Sur Global, nos plantean realidades que, salvo escasas excepciones y proyectos aislados, han sido poco consideradas en las políticas públicas de vivienda de los países de la región, sin embargo, constituyen experiencias necesarias para escribir un siguiente capítulo sobre el hábitat desde el Sur.

\section{Palabras clave}

Hábitat; Arquitectura; Derecho a la Ciudad; Participación Social; Sur Global

1 Arquitecta especialista en planeación urbana, movilidad cotidiana y espacio público. Instituto de investigación y Estudios de las Ciudades, Universidad de Guadalajara. Calzada Independencia Norte 5075, Huentitán el Bajo, Guadalajara, Jalisco, México. E-mail: ines.olivares@académicos.udg.mx 


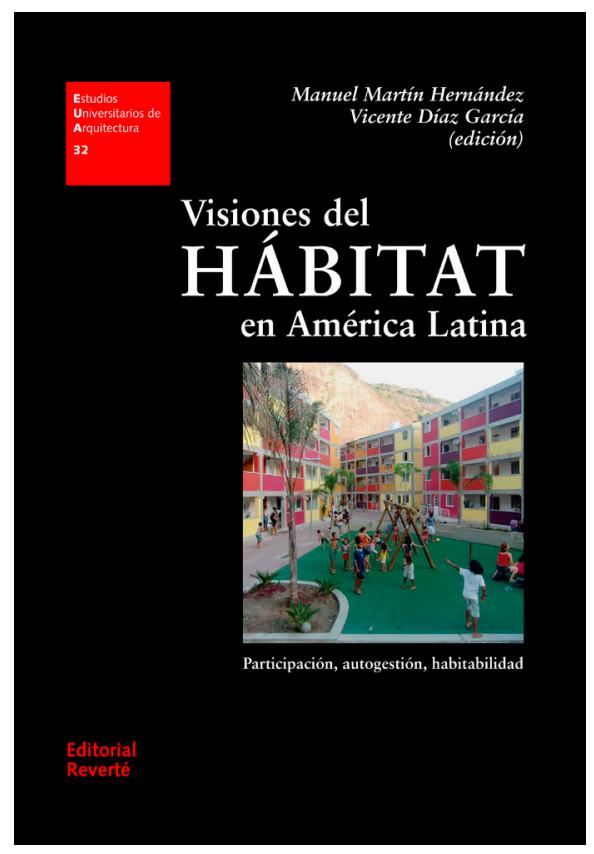

Martín Hernández, Manuel y Díaz García, Vicente (eds.) (2018). Visiones del hábitat en América Latina. Participación, autogestión, habitabilidad. Barcelona: Editorial Reverté. ISBN 978-84-291$2132-2$
Este es un libro que desafía los paradigmas actuales sobre el hábitat. Desafía también las estigmatizaciones sobre las prácticas sociales que se desarrollan sobre el hábitat en los países y ciudades del sur global. Asimismo, desafía tratar el hábitat tomando como eje una disciplina sobre la que no es tendencia reflexionar críticamente: la arquitectura. Es precisamente en estos desafíos donde se centra el valor del libro $V i$ siones del Hábitat en América Latina editado por Manuel Martin Hernández y Vicente Díaz, en el que participan intelectuales, al mismo tiempo activistas, de larga trayectoria en la reflexión de las diferentes realidades que constituyen el hábitat en la región. Celebramos la iniciativa del prestigiado grupo editorial Reverté de voltear a ver otras perspectivas de lo que sucede en América Latina, en su línea editorial de arquitectura, en una temática que refresca los enfoques tradicionales del hábitat y hace aportaciones desde el Sur a todos los países inmersos en el capitalismo global, que presenta en la materialidad arquitectónica de la vivienda, las expresiones más nítidas de la desigualdad.

Por otro lado, este libro pone en perspectiva la importante tarea que han realizado personas e instituciones como el Programa Iberoamericano de Ciencia y Tecnología para el Desarrollo (CYTED) que, en 1987 con la creación del subprograma más tarde denominado HABYTED, aportó el primer eslabón para generar una red multidisciplinaria de especialistas en el estudio del hábitat para los sectores sociales de menores recursos de Iberoamérica. A partir de tres estrategias: proyectos de investigación, proyectos de innovación y redes temáticas; HABYTED desarrolló hasta principios del siglo xx diversas aportaciones que han dejado importantes avances respecto de la teoría y producción del hábitat (Romero y Mesías, 2004).

El territorio de América Latina, extenso apenas en poco más de 21 millones de kilómetros cuadrados en el que residen más de 500 millones de habitantes y donde conviven una amplia diversidad de realidades, es el centro de la reflexión.

Los prólogos y prefacio forman también parte del desafío que encarna el libro en su conjunto. Así, desde un prólogo que se niega como tal y se afirma manifiesto, Raquel Rolnik, autora del libro La Guerra de los lugares: la colonización de la tierra y de la vivienda en la era de las finanzas, denuncia la "financiarización" de la vivienda por el capital financiero global, como un nuevo imperio colonial "sin bandera ni rostro". Las expresiones materiales y sociales de este imperio pueden ser más o menos particulares y profundas, de acuerdo a las normativas, capacidades técnicas y ética política del Estado y de las entidades de planificación en cada ciudad, las cuales son cobijadas por el paradigma de la propiedad individual del suelo sobre otras formas de relación -ilegales en general- de los individuos con el territorio.

Por su parte, Josep María Montaner destaca la amplia experiencia en investigación, critica y construcción del hábitat en la región latinoamericana y rescata del libro la idea de que el hábitat popular y la participación no están reñidos con la calidad arquitectónica y urbana, con relación a la calidad constructiva y el acceso a infraestructuras urbanas necesarias para la vida. Así, según Montaner, la arquitectura y el urbanismo más astutos y avanzados han podido dar respuesta, en experiencias muy concretas, al hábitat popular en América Latina.

Manuel Martín Hernández y Vicente Díaz García, a partir de la cooperación y solidaridad que desde la dictadura franquista han tenido España y América Latina, hacen un recuento histórico de los eventos, oficiales y alternativos, así como de las personas que, como profesores universi- 
tarios, investigadores especializados o activistas sociales, han cimentado el largo andar que tiene ya el análisis crítico y la construcción del hábitat popular en la región. Concluyen, en su prefacio, la necesidad de redoblar esfuerzos para producir un cambio, ya que a pesar de todo el trabajo desarrollado hasta ahora aún no ha sido posible incorporar estos aportes a las disciplinas arquitectónicas y urbanas de la región.

\section{¿El despertar de América Latina? Ya estamos despiertos, pero aún no somos escuchados}

El corpus del libro esta integrado por quince capítulos de los cuales nueve hacen reflexiones teóricas en torno a problemas de la producción del hábitat en la región; y otros consideran oportuno tomar estudios de caso como metodología para plantear problemas y alternativas posibles a la construcción del hábitat.

En la primera clasificación los autores nos sitúan en la necesidad de construir una visión del hábitat desde el contexto y experiencias del Sur, en particular desde las acciones que la población empobrecida realiza para gestionar su hábitat. Desde esta perspectiva plantean la importancia de replantearnos el "derecho a la ciudad", mas allá de las manipulaciones del concepto por los organismos internacionales e instituciones locales; así como superar el también manipulado concepto de "participación ciudadana" por el de "integración social" que implica la participación plena del usuario en el proceso de producción del hábitat. En la segunda clasificación los estudios de caso constituyen ámbitos urbanos que permiten observar los procesos que denuncian nuevas formas de apropiación del territorio por el capital (Ciudad de México, Argentina) o alternativas positivas a los problemas del hábitat (Bogotá y Medellín en Colombia, El Salvador, Uruguay y Chile).

Manuel Martín y Vicente Díaz plantean la importancia de hablar de "Otros hábitats en América Latina" como una postura contra la colonización intelectual del pensamiento europeo y anglosajón en las disciplinas vinculadas a la construcción del hábitat, tanto en las instituciones educativas como en los diferentes niveles de gobierno responsables de su gestión. Así, cuando se refieren a "otros hábitats" plantean la importancia de las arquitecturas que están fuera de la excepcionalidad, las que constituyen el espacio de vida de la mayor parte de la población en las ciudades, en las que el autor deja de ser el centro de la preocupación del proyecto, y por esta circunstancia son actualmente las de mayor interés para el futuro de las ciudades. Para los autores, la búsqueda de la habitabilidad y de su gestión democrática constituye el mayor reto de la humanidad que vive en las ciudades, especialmente aquellas localizadas en los mal llamados "países en vías de desarrollo", que al definirlas como "impredecibles, indeterminadas y cambiantes" constituyen el paradigma de la ciudad posmoderna.

Desde una perspectiva que aborda los diferentes procesos globales en los que se insertan las ciudades, Daniel González denuncia la manipulación de la idea del "derecho a la ciudad" y de otros paradigmas subyacentes a este con diferentes "claves interpretativas", para articular imaginarios, ficciones y utopías, algunas veces bienintencionadas, pero la mayor parte como sustento de nuevas formas de apropiación del territorio por el capital. Convoca, desde esta perspectiva, a la revisión de esta utopía que obliga a entender a la ciudad como espacio político sede por excelencia del conflicto emanado de la lucha de clases. 
A partir de su vasta experiencia como estudioso y consultor en materia de vivienda, Victor Pelli propone situar a la "integración social" como eje central de las políticas habitacionales en América Latina y superar la idea de "participación" que forma parte de la postura asistencialista sustentada en dotar a la población empobrecida de ciertos bienes y servicios, establecidos como necesarios por el estado y los dueños del capital, sin considerar a los beneficiarios genuinos en el proceso. Mediante una lúcida reflexión sobre las carencias que caracterizan a la pobreza urbana, demuestra que estas van mas allá de la supervivencia física y "frenan la evolución de nuestras sociedades hacia una estructura de relaciones igualitarias y equitativas" (ob. cit., p. 64).

Joan MacDonald, establece la necesidad de poner en el eje de los estudios sobre el hábitat latinoamericano a los propios habitantes de los tugurios, a los pobres urbanos que por décadas han hecho incansables esfuerzos por mejorar las condiciones de sus espacios de vida cotidiana. A partir de nueve temas que sustentan su postura, la autora expone su amplio conocimiento como presidenta del Servicio Latinoamericano, africano y Asiático de Vivienda Popular (SELAVIP) y convoca a los investigadores a profundizar en dichos procesos.

Ana Sugranyes hace una revisión del concepto de "habitabilidad" bajo la premisa de que la mercantilización del suelo, la vivienda y el espacio público, es decir, los componentes más importantes del hábitat en las ciudades, requieren ser retomados a partir de los valores vinculados a la "dignidad humana". Para aclarar su argumentación toma como caso el fenómeno de "Los con techo" en Chile, es decir, la población residente de viviendas con subsidio habitacional, política estatal que nace a mediados del siglo xx que, si bien ha logrado disminuir el déficit habitacional, también ha perpetuado la pobreza como forma de vida. A partir de esta denuncia hace un "[...] llamamiento al rescate de los derechos humanos [...]" (ob. cit., p. 51).

Julián Salas y Belén Gesto ponen el centro de su reflexión en "la mejora de los barrios precarios como antídoto a la violencia urbana". Las cifras que presentan los autores muestran, sin lugar a duda, que la violencia urbana es un problema en crecimiento en el conjunto de la región latinoamericana de manera que bajo su concepción como una conducta antisocial y bajo la premisa de su relación estrecha con la pobreza urbana y la exclusión social, exponen que cambiar el ambiente físico puede ser un medio poderoso para reducir el crimen. A partir de dos casos en El Salvador y Bogotá (Colombia) muestran las potencialidades de su argumentación.

Desde su experiencia en la docencia, la investigación universitaria y el activismo social, Esteban de Manuel Jerez expone la construcción de un triángulo relacional entre la enseñanza de la arquitectura, el dibujo de arquitectura y la arquitectura misma; y pone este triangulo a su vez en interrelación con el triángulo de la función social de la universidad, integrado por las actividades de extensión, investigación y docencia, como la base de sus aportaciones. Desde esta visión, analiza en tres apartados clave para el autor la relación entre la universidad y la sociedad, la transformación social de la universidad y la contribución de la universidad para la transformación social del hábitat, para luego analizar su aplicación en dos casos: la consolidación del asentamiento de Jnane Aztout en Marruecos y la rehabilitación integral del barrio de Alcosa en Sevilla, España.

El "diseño participativo" es abordado por Gustavo Romero, José Salceda, Javier Hernández y Ulises Castañeda, integrantes del grupo de in- 
vestigación Arquitectura, Diseño, Complejidad y Contradicción de la UNAM, en su relación intrínseca con la "producción social del hábitat”, ya que implica poner el acento en los sujetos y sus necesidades más que en el objeto mismo, la vivienda, que históricamente ha sido el eje de las políticas públicas en la región. Bajo la premisa de que "todo ser humano tiene una percepción y un conocimiento de lo que llamamos arquitectura" (p. 125), los autores convocan a una "producción social del hábitat asistida" (PSHA), es decir, a la participación conjunta entre habitantes y otros actores implicados en el proceso definido como un "derecho" desde una postura ideológico-política y no como una opción que puede o no dar el Estado. Así, el "diseño participativo" se propone como un "medio de participación plena, consciente e informada de todos los actores en una deliberación consensuada" (p. 127), el cual ejemplifican en el conjunto Canal Nacional en la Ciudad de México.

Jorge M. Jáuregui, a partir de su experiencia en el estudio e intervención de las favelas de Río de Janeiro y desde una visión de la arquitectura que integra los aspectos objetivos, vinculados al dominio técnico y la materialización de ideas, y subjetivos, vinculados a los valores espirituales, considera necesaria la actitud analítica, experimental y autocrítica para asumir los desafíos de la ciudad. Propone poner a la "habitación" como el centro de las preocupaciones sociales, en el contexto de una triada formada por el medio ambiente, la desigualdad y la movilidad, que constituyen la cuestión urbana de las ciudades del siglo xxI. Así, urbanizar favelas implica introducir la lógica en el caos en donde el "proyecto arquitectónico y urbano, constituye "una apuesta lanzada [...] al futuro e implica asumir los desafíos [...]" (p. 149), en donde la articulación entre la ética, la estética y la política resultan significativas en la búsqueda de integrar a las diferencias.

Carlos González Lobo pone su atención en el problema de acceso a la "vivienda necesaria y conveniente" en la Ciudad de México, específicamente, en donde considera necesaria la articulación entre los sectores publico, privado, instituciones académicas y organizaciones de la sociedad civil. Mediante un análisis histórico contextual el autor arriba al siglo XxI dibujando un escenario formado por conjuntos de vivienda de interés social y de origen irregular - la solución popular-, sumadas a cinco millones de viviendas de construcción reciente, abandonadas y vandalizadas, producto del mercado neoliberal. El autor analiza, a partir de la "necesidad y la conveniencia", la tipología de la vivienda de interés social, la ausencia de propuestas para la población que esta fuera de dicho mercado, así como las soluciones emergentes que los propios habitantes han dado para resolver parcialmente el problema de acceso al suelo.

Bernabela Pelli y Ángeles D’Aveta centran su reflexión en los procesos de participación en la construcción del hábitat en Argentina a partir del Programa Integral de Mejoramiento de Barrios (ProMeBa 20082015) y el estudio del caso de la ciudad de Resistencia. Entre sus hallazgos destacan que los procesos de participación están muy vinculados a las trayectorias y experiencias previas de los vecinos en la autogestión de su hábitat, y que los aprendizajes que adquieren las comunidades, con independencia de los resultados, se constituye en herramientas de empoderamiento y visibilización en los que las tecnologías de la información y la comunicación juegan un rol fundamental como facilitadores de la participación. Así, las autoras proponen cambiar el paradigma del "sujeto de necesidades" al "sujeto de deseos" que sitúe al habitante como un semejante. 
A partir de su trayectoria en importantes cargos de planeación urbana en Colombia y Medellín, Jorge Pérez Jaramillo nos introduce en la complejidad de los problemas sociales y urbanos que posicionaron a esta ciudad como una de las más conflictivas del mundo, y que desde los años 80 inició un proceso de transformación estructural movido por un amplio despliegue participativo que situó entre sus objetivos la corresponsabilidad y el fortalecimiento de la institucionalidad, la cultura y la educación. En este contexto plantea la problemática y debate que ha representado el río Aburrá-Medellín, al cual sitúa al mismo tiempo como una oportunidad de redensificar mediante el proyecto metropolitano Parques del Río, desde una perspectiva ecológicamente responsable, socialmente incluyente y económicamente viable. La suspensión del proyecto pone en evidencia el "olvido" de la historia urbana reciente que fue la base para "honrar el proyecto ciudadano sin mesianismos, como una construcción social y política de todos" (ob. cit., p. 185).

Héctor Gaete, posiciona su análisis en las actuaciones públicas de vivienda en Chile como resultado del sismo y tsunami de febrero del 2010 y el papel del Estado, en tanto responsable de la provisión de vivienda social. Así, contextualiza a Chile como un estado neoliberal convertido en mecanismo de reproducción de desigualdades, sobretodo derivadas de políticas públicas de acceso al suelo, que en manos de la iniciativa privada habían condenado a los pobres a residir en las periferias en condiciones de baja habitabilidad. En razón del impacto causado por el sismo y tsunami en alrededor de 370000 viviendas destruidas y un Estado incapaz de resolver a la emergencia, se inició un programa de reconstrucción de viviendas mediante cuatro tipologías acordes con su localización, densidad y agrupación. Así, quedo patente que la coordinación del Estado es crucial para resolver emergencias de gran envergadura con apoyo de la iniciativa privada, cambiando el rumbo del Ministerio de Vivienda y Urbanismo (MIMVU), cuyos objetivos ahora están centrados en "posibilitar el acceso a soluciones habitacionales de calidad, principalmente a los sectores vulnerables, emergentes y medios, bajo criterios de pertinencia equidad y participación" (ob. cit., p. 198).

Aurelio Ferrero, Laura Fleker y Gustavo Pelegrin, desde su experiencia como director y becarios en el Centro Experimental de la Vivienda Económica (CEVE), Argentina, se centran en los alcances y limitaciones de la tecnología en la cuestión habitacional. Concluyen en su reflexión que la tecnología por si misma no puede ofrecer soluciones si estas no se insertan en un modelo de gestión que considere la caracterización multidimensional del problema de la vivienda, así como la participación y posicionamiento de actores que estén dispuestos a invertir recursos para apoyar su resolución. Proponen, a manera de conclusión, tres criterios para reflexionar sobre el cambio de contexto que afecta la pertinencia del CEVE, al que agregaríamos el conjunto de las instituciones orientadas al estudio y producción del hábitat: la repetición de experiencias previas para resolver los problemas del hábitat; la articulación de actores y recursos para garantizar la sostenibilidad de hábitat; y la capacidad de las instituciones de ciencia y tecnología para construir puentes interdisciplinarios y la participación social.

Benjamín Nahúm y Raúl Vallés hacen una reflexión sobre la historia del cooperativismo en Uruguay como instrumento central de la política de vivienda con el que han sido beneficiadas miles de familias de todos los niveles socioeconómicos. Los autores definen como esencia del 
cooperativismo de vivienda los siguientes aspectos: la solidaridad social en la construcción y el financiamiento de las viviendas; la participación organizada en asambleas; la autogestión en la toma de decisiones; el esfuerzo propio a partir de las capacidades individuales; y la propiedad colectiva de la vivienda. Suman a dichos aspectos la necesaria participación del Estado a partir del financiamiento público y el apoyo a los Institutos de Asistencia Técnica. Concluyen que la importancia del sistema cooperativo uruguayo se sustenta en entender el "habitar" como una propuesta "comunitaria" que "[...] genera verdaderas escuelas de ciudadanía” (ob. cit., p. 224).

\section{Llamamiento}

Los "territorios del neoliberalismo", como denominó Pradilla Cobos (2009) a los impactos territoriales del patrón neoliberal de acumulación del capital, se observan con mayor nitidez en los procesos oficiales de producción del hábitat orientado a los sectores de menores recursos, en su localización periférica y en su baja accesibilidad urbana, en las deficiencias de su infraestructura básica, en la baja calidad de los materiales constructivos, en los escasos metros de superficie habitable; paisajes que superan ficciones urbanas postmodernas que vaticinaban la catástrofe de la vida en las grandes ciudades como Metrópolis de Fritz Lang (Pommer, 1927) donde la "ciudad máquina" funciona a partir del proletariado que habita en guetos subterráneos o El Proceso, de Orson Welles (Salkind, 1962), donde se muestran edificios que congregan a la población trabajadora, mediante tipologías de colmena que responden a los objetivos de control y productividad (Ocaranza Velasco, 2016).

Este documento constituye un llamamiento a los profesionales de la arquitectura, a los arquitectos en formación y, en general, a los profesionales involucrados en la gestión del hábitat, para que tomemos las experiencias de este escogido grupo de autores como punto de partida para plantear nuevas preguntas, respuestas y soluciones a los problemas de hábitat en el Sur desde el Sur. Esto no significa "no mirar" hacia otras realidades, significa hacerlo desde la reflexión crítica, para construir nuevas ficciones urbanas. 


\section{Bibliografía}

Ocaranza Velasco, Jorge Octavio (2016). Ciudades cyberpunk en el imaginario posmoderno. Aproximación semiótica-simbólica de narrativas de imagen cinemática del paisaje de ciudad (Tesis doctoral). Universidad de Guadalajara, Guadalajara, México.

Pommer, Erich (Productor), von Harbou, Thea (Escritor), \& Lang, Fritz (Director) (1927). Métropolis [Película]. República de Weimar: UFA.

Pradilla Cobos, Emilio (2009). Los territorios del neoliberalismo en América Latina. Compilación de ensayos. México D.F.: UAM.
Rolnik, Raquel (2015). La Guerra de los lugares: la colonización de la tierra y de la vivienda en la era de las finanzas. Santiago de Chile: LOM Ediciones

Romero, Gustavo, y Mesías, Rosendo (eds.) (2004). La participación en el diseño urbano y arquitectónico en la producción social del hábitat. México D.F.: CYTEDHABYTED.

Salkind, Alexander (Productor), Welles, Orson y Cholot, Pierre (Adaptación), y Wells, Orson (Director) (1962). El Proceso [Película]. Francia/ Alemania/ Italia: FICIT.

Olivares González, Adriana Inés (2019). ¿Por qué pensar sobre el hábitat desde la arquitectura en el sur y desde el sur global? Hábitat y Sociedad, 12, 115-122.

<http://dx.doi.org/10.12795/HabitatySociedad.2019.i12.07> 